\title{
Cyclin-dependent kinase subunit 2 overexpression promotes tumor progression and predicts poor prognosis in uterine leiomyosarcoma
}

\author{
YUPENG DENG $^{1,2^{*}}$, QUN HAN $^{3 *}$, SHUYU MEI $^{1}$, HAILING LI $^{1}$, FAN YANG $^{1,4}$, JUN WANG $^{1}$, \\ SHUANG GE ${ }^{1}$, XIAOTONG JING ${ }^{1}$, HUI XU ${ }^{1}$ and TINGGUO ZHANG ${ }^{1}$
}

\author{
${ }^{1}$ Institute of Pathology and Pathophysiology, Shandong University School of Medicine, Jinan, Shandong 250012; \\ ${ }^{2}$ Department of Pathology, The Second Hospital of Shandong University, Jinan, Shandong 250033; ${ }^{3}$ Department of Pathology, \\ Qilu Hospital of Shandong University, Qingdao, Shandong 266035; ${ }^{4}$ Clinical Center of Gene and Cell Engineering, \\ Beijing Shijitan Hospital, Capital Medical University, Beijing 100038, P.R. China
}

Received November 14, 2018; Accepted May 22, 2019

DOI: $10.3892 / \mathrm{ol} .2019 .10668$

\begin{abstract}
Cyclin-dependent kinase subunit(CKS) 2 is a member of the CKS family, which plays an important role in the regulation of meiosis and mitosis. Overexpression of CKS2 has been reported in several types of tumors. However, few studies have investigated its role in uterine leiomyosarcoma (ULMS). In the present study, the expression of CKS2 in 38 cases of ULMS and 38 cases of uterine leiomyoma (ULM) was analyzed by immunohistochemistry. Moreover, the functional analysis of CKS2 was performed in ULMS cell lines. A significantly higher expression of CKS2 was found in ULMS tissues than in ULM tissues $(\mathrm{P}<0.01)$ and high CKS2 expression was associated with increased tumor size, low progesterone receptor expression and poor prognosis in patients with ULMS. Multivariate Cox regression analysis revealed that CKS2 expression status was an independent predictor of overall survival for ULMS. Furthermore, silencing of CKS2 in ULMS cells inhibited cell proliferation, colony formation, migration and invasion, and resulted in cell cycle arrest. In conclusion, the present study demonstrated that CKS2 may serve as a marker for the differential diagnosis of ULMS and ULM. In addition, it may act as an independent prognostic factor in patients with ULMS, and serve as a novel target for ULMS therapy.
\end{abstract}

Correspondence to: Professor Tingguo Zhang, Institute of Pathology and Pathophysiology, Shandong University School of Medicine, 44 Wenhuaxi Road, Jinan, Shandong 250012, P.R. China E-mail: ztguo@sdu.edu.cn

*Contributed equally

Abbreviations: CKS2, cyclin-dependent kinase subunit 2; ULMS, uterine leiomyosarcoma; ULM, uterine leiomyoma; siRNA, small interfering RNA; PR, progesterone receptor

Key words: cyclin-dependent kinase subunit 2, uterine leiomyosarcoma, prognosis, proliferation, invasion

\section{Introduction}

Uterine sarcomas represent $\sim 8 \%$ of uterine malignant tumors and entail a high mortality rate, with an overall 5-year survival rate of $\sim 30 \%$ (1). Uterine leiomyosarcoma (ULMS) is the most common uterine sarcoma, characterized by poor prognosis, early metastasis and high rate of recurrence (2-4). ULMS occurs mostly in women over the age of 40 , and its symptoms include abnormal vaginal bleeding, palpable pelvic mass and pelvic pain (5). These features are similar to the symptoms of uterine leiomyoma (ULM) and therefore it is difficult to distinguish ULMS from ULM prior to surgery. Currently, there is still no optimal treatment for ULMS $(5,6)$. There are few studies on ULMS and little is known about its associated oncogenic pathways.

The human CKS family consists of two members, CKS1 and CKS2, which share $>80 \%$ sequence identity (7). CKS1 is a specific cofactor that is necessary for the degradation and ubiquitination of p27 by the Skp1-Cullin-F-box protein complex of S-phase kinase-associated protein 2 (8). CKS2 is also important during early embryonic development, for the process of somatic cell division (9). In addition, it has been shown to be essential for the first metaphase/anaphase transition of mammalian meiosis (10). Several reports have indicated that CKS2 was upregulated in a number of types of tumors, including breast cancer, esophageal carcinoma, gastric cancer, colorectal cancer, hepatocellular carcinoma, cholangiocarcinoma and bladder cancer (11-17). However, the underlying cellular functions of CKS2 and the associated mechanism involved in its carcinogenicity remain unclear.

To identify a candidate gene that may contribute to the progression of ULMS, a search in the Gene Expression Omnibus (GEO) database was conducted. Expression arrays from the GEO datasets (GSE64763, GSE764 and GSE36610) showed distinct levels of expression in ULMS and ULM of genes such as cyclin-dependent kinase subunit (CKS)2, thymidylate synthase and putative tenascin-XA. Since CKS2 was found to be expressed in several tumors and little was known about its role in ULMS, further analyses were conducted. The 
present study investigated CKS2 expression in ULM/ULMS and determined the role of CKS2 in the development of ULMS.

\section{Materials and methods}

Clinical tissue samples. The specimens of 38 cases with ULMS and 38 cases with ULM were collected between January 2005 and October 2015 at Qi Lu Hospital of Shandong University (Jinan, China). At the beginning of the study, 45 cases with ULMS were recruited and followed-up over the telephone. Seven cases were lost during the follow-up and 38 remained, with the complete clinical and prognostic information. By the end of the follow-up (March 2016), 19 patients with ULMS were deceased. The median age of the patients with ULMS was 45 years (range, 29-76 years). The median age of the patients with ULM was 38 years (range, 25-65 years). None of these patients had received pre-operative chemotherapy or radiotherapy. All patients provided informed consent, and the study was approved by the Ethics Committee of Shandong University (approval no. 201302036). All samples were assessed by two well-trained pathologists. The diagnosis was confirmed according to the 2003 World Health Organization criteria (18), and pathological records were reviewed according to the 2009 International Federation of Obstetrics and Gynecology (FIGO) staging for sarcomas (19).

Immunohistochemistry (IHC). Tissues were fixed with formalin $(10 \%, \mathrm{pH} 7.2)$ at room temperature for $24 \mathrm{~h}$ and then embedded in paraffin. IHC analysis was performed on these formalin-fixed, paraffin-embedded specimens ( $4-\mu \mathrm{m}$ sections). The sections were rehydrated through alcohol gradient as follows: Anhydrous ethanol I for $5 \mathrm{~min}$; anhydrous ethanol II for $5 \mathrm{~min}$; $95 \%$ ethanol for $5 \mathrm{~min}$; $85 \%$ ethanol for $5 \mathrm{~min}$; and $75 \%$ ethanol for $5 \mathrm{~min}$. The sections were submerged in sodium citrate buffer at $100^{\circ} \mathrm{C}$ for $5 \mathrm{~min}$ for antigenic retrieval. The endogenous peroxidase activity was blocked with $3 \%$ hydrogen peroxide for $10 \mathrm{~min}$ at room temperature. After blocking, the sections were incubated with anti-CKS2 primary antibody (1:100; cat. no. ab155078; Abcam) overnight at $4^{\circ} \mathrm{C}$. A PV-9000 2-step plus ${ }^{\circledR}$ Poly-HRP Anti-Mouse/Rabbit IgG Detection System (Zhongshan Golden Bridge Biotechnology Company) was used for immunohistochemical assay according to the manufacturer's instructions. PBS was used as the negative control.

Values were assigned to the samples according to the percentage of positive cells: $0, \leq 5 ; 1,6-25 ; 2,26-50 ; 3,51-75$; and $4,>75 \%$. Similarly, the staining intensity scores were assigned as follows: 0 , no staining; 1 , weak; 2 , moderate; and 3 , strong staining. The product of the two integers was used to divide the participants into two groups. Participants with a product $\geq 4$ were designated to the 'high expression' group, and those with a product $<4$ were designated to the 'low expression' group. The images were captured with an Olympus BX53 optical microscope (Olympus Corporation) at X400 magnification. Two senior pathologists independently assessed these scores.

Cell culture and transfection. Human ULMS SK-UT-1 and SK-UT-1B cell lines were kindly provided by Dr Kong Beihua of the Qi Lu Hospital of Shandong University. These cell lines originate from the uterus and have been reported to differ markedly in terms of morphology and karyotype (20). The cells were maintained in minimal essential medium (MEM; cat. no. 11095080; Gibco; Thermo Fisher Scientific, Inc.), supplemented with $10 \%$ fetal bovine serum (FBS; Biological Industries), 1X solution of non-essential amino acids (Thermo Fisher Scientific, Inc.), $1 \mathrm{mM}$ sodium pyruvate and $100 \mathrm{U} / \mathrm{ml}$ penicillin and streptomycin in $5 \% \mathrm{CO}_{2}$ at $37^{\circ} \mathrm{C}$.

Small interfering RNA (siRNA) targeting CKS2 (si-CKS2 sense, 5'-GGAGACUUGGUGUCCAACATT-3'; and si-CKS2 antisense, 5'-UGUUGGACACCAAGUCUCCTC-3') and negative control siRNA (si-Ctrl sense, 5'-UUCUCCGAACGUGUC ACGUTT-3'; and si-Ctrl antisense, 5'-ACGUGACACGUU CGGAGAATT-3') were obtained from Shanghai GenePharma Co., Ltd. The siRNA oligomer was diluted in Opti-MEM (Thermo Fisher Scientific, Inc.). The diluted siRNA oligomer was mixed with diluted Lipofectamine ${ }^{\circledR} 2000$ (Thermo Fisher Scientific, Inc.) and incubated for $20 \mathrm{~min}$ at room temperature to allow the siRNA-lipid complexes to form. The complexes were then added to each well of 6-well plates, giving a final concentration of siRNA of $50 \mathrm{pmol} / \mathrm{ml}$. After incubation in $5 \% \mathrm{CO}_{2}$ at $37^{\circ} \mathrm{C}$ for $4-6 \mathrm{~h}$, the cells were washed three times with Opti-MEM, and returned for incubation for the following procedures.

Cell Counting Kit-8 (CCK-8) cell proliferation assay. Cell proliferation rates were measured using the CCK-8 (Beyotime Institute of Biotechnology). Following the transfection of cells with si-CKS2 and si-Ctrl (after $24 \mathrm{~h}$ ), the cells were seeded at a density of $3 \times 10^{3}$ per well in a 96 -well plate. The cells were incubated for $24,48,72$ or $96 \mathrm{~h}$ and $10 \mu \mathrm{l}$ CCK-8 was subsequently added into each well. The absorbance was measured at $450 \mathrm{~nm}$ by a microplate reader (Bio-Rad Laboratories, Inc.), following incubation in $5 \% \mathrm{CO}_{2}$ at $37^{\circ} \mathrm{C}$ for $2 \mathrm{~h}$.

Colony formation. Following $24 \mathrm{~h}$ from transfection with si-CKS2 or si-Ctrl, SK-UT-1 or SK-UT-1B cells ( 500 cells/well) were seeded in 6-well plates and incubated for 14 days to form colonies. The colonies were fixed with $4 \%$ paraformaldehyde at room temperature for $20 \mathrm{~min}$ and stained with $0.1 \%$ crystal violet at room temperature for $20 \mathrm{~min}$. Images of colonies were captured by a digital camera and the number of foci containing $>50$ cells was counted. The mean number of foci formed by si-Ctrl was normalized to $100 \%$ and compared with the number of si-CKS2 colonies was compared with it.

Cell migration and invasion assays. The migration assays were carried out using Transwell inserts (Corning Inc.), according to the manufacturer's instructions. For the invasion assays, the membranes of Transwell inserts were coated with Matrigel matrix (BD Biosciences). The Matrigel matrix was diluted by Opti-MEM (1:3; Thermo Fisher Scientific, Inc.) and incubated at $37^{\circ} \mathrm{C}$ for $3 \mathrm{~h}$ to form the Matrigel coating. Following $24 \mathrm{~h}$ transfection with si-RNA, cells $\left(1 \times 10^{5}\right)$ in $100 \mu \mathrm{l}$ serum-free medium were placed in the upper chamber, and the lower chamber was filled with $700 \mu \mathrm{l}$ culture medium with $10 \%$ FBS. The cells were incubated for $24 \mathrm{~h}$ at $37^{\circ} \mathrm{C}$. The non-migrated/-invaded cells that remained on the upper chamber were removed. The cells on the lower side of the Transwell membrane were fixed with $4 \%$ paraformaldehyde at room temperature for $20 \mathrm{~min}$ and stained with $0.1 \%$ crystal 
violet at room temperature for $20 \mathrm{~min}$. The number of migrated and invaded cells was counted under a light microscope at 200x magnification in six random fields.

Cell cycle. The effects of silencing CKS2 on cell cycle progression were assessed using propidium iodide staining and analyzed by flow cytometry. Cells were seeded in 6-well plates $\left(2 \times 10^{5}\right.$ per well) and transfected with si-CKS2 and si-Ctrl. After $48 \mathrm{~h}$, cells were washed with PBS, harvested and fixed in $70 \%$ ethanol at $4^{\circ} \mathrm{C}$ overnight. Cells were treated with DNase-free RNase and stained with propidium iodide (400 $\mu 1$ from $50 \mu \mathrm{g} / \mathrm{ml}$ stock solution) at $4^{\circ} \mathrm{C}$ for $30 \mathrm{~min}$, and the cell cycle was analyzed by flow cytometry based on the DNA content of cell populations. Finally, the distribution of cells within the $G_{0} / G_{1}, S$, and $G_{2} / M$ phases was measured by using ModFit LT 5.0 (Verity Software House, Inc.).

Western blot analysis. Following $48 \mathrm{~h}$ transfection with si-RNA, total protein was extracted from the sample with RIPA lysis buffer and protein concentrations were detected by the BCA protein assay kit (both from Beyotime Institute of Biotechnology). Equal amounts of protein $(30 \mu \mathrm{g})$ were applied to $15 \%$ sodium dodecyl sulfate polyacrylamide gel electrophoresis gel and electroblotted to a $0.22-\mu \mathrm{m}$ polyvinylidene difluoride membrane. The membranes were blocked by QuickBlock $^{\mathrm{TM}}$ Blocking Buffer for Western Blot (Beyotime Institute of Biotechnology) at room temperature for $10 \mathrm{~min}$. After the blocking step, the membranes were incubated with rabbit anti-human CKS2 monoclonal antibody $(1: 1,000$; cat. no. ab155078; Abcam), anti-beta actin antibody (1:1,000; cat. no. ab8227; Abcam), anti-claudin-1 antibody (1:1,000; cat. no. ab15098; Abcam), anti-p38 antibody $(1: 1,000$; cat. no. ab195049; Abcam) and anti-bax antibody (1:1,000; cat. no. ab32503; Abcam) at $4^{\circ} \mathrm{C}$ overnight and then with horseradish peroxidase-labeled goat anti-rabbit $\operatorname{IgG}(1: 5,000$; cat. no. ab 6721; Abcam) at $37^{\circ} \mathrm{C}$ for $30 \mathrm{~min}$. Finally, the membranes were analyzed using an electrochemiluminescence system (EMD Millipore).

Reverse transcription-quantitative PCR ( $q P C R)$. Following $24 \mathrm{~h}$ transfection with si-RNA, total RNA was extracted from cells using TRIzol reagent (Thermo Fisher Scientific, Inc.) and was then transcribed into cDNA with ReverTra Ace qPCR RT kit (cat. no. FSQ-101; Toyobo Life Science). The thermocycling conditions were as follows: $37^{\circ} \mathrm{C}$ for $15 \mathrm{~min}$ and $98^{\circ} \mathrm{C}$ for 5 min. Quantification of the cDNA template was performed on a Real-time Thermo Cycler (cat. no. C1000; Bio-Rad Laboratories, Inc.), using SYBR-Green Real-time PCR Master mix (cat. no. QPK-201; Toyobo Life Science). The thermocycling conditions were as follows: Initial denaturation at $95^{\circ} \mathrm{C}$ for $10 \mathrm{~min}$, and 40 cycles of denaturation at $95^{\circ} \mathrm{C}$ for $10 \mathrm{sec}$, annealing at $60^{\circ} \mathrm{C}$ for $1 \mathrm{~min}$, and extension at $65^{\circ} \mathrm{C}$ for $5 \mathrm{sec}$. The primers were as follows: CKS2 forward, 5'-TTCGAC GAACACTACGAGTACC-3'; CKS2 reverse, 5'-GGACAC CAAGTCTCCTCCAC-3'; GAPDH forward: 5'-TGAAGG TCGGAGTCAACGGA-3'; and GAPDH reverse, 5'-CCTGGA AGATGGTGATGGGAT-3'. The dissociation curve analysis was performed in order to guarantee the specificity of the qPCR. The relative expression level of CKS2 was normalized to GAPDH and analyzed by the $2^{-\Delta \Delta \mathrm{Ct}}$ method (21).
Table I. Expression of CKS2 in ULMS and ULM tissues.

\begin{tabular}{lccc}
\hline & \multicolumn{2}{c}{ CKS2 expression } & \\
\cline { 2 - 3 } Tissue & High, $\mathrm{n}$ & Low, $\mathrm{n}$ & P-value \\
\hline Tissue type & & & $<0.001$ \\
ULMS & 24 & 14 & \\
ULM & 7 & 31 & \\
\hline
\end{tabular}

CKS2, cyclin-dependent kinases subunit 2; ULMS, uterine leiomyosarcoma; ULM, uterine leiomyoma.
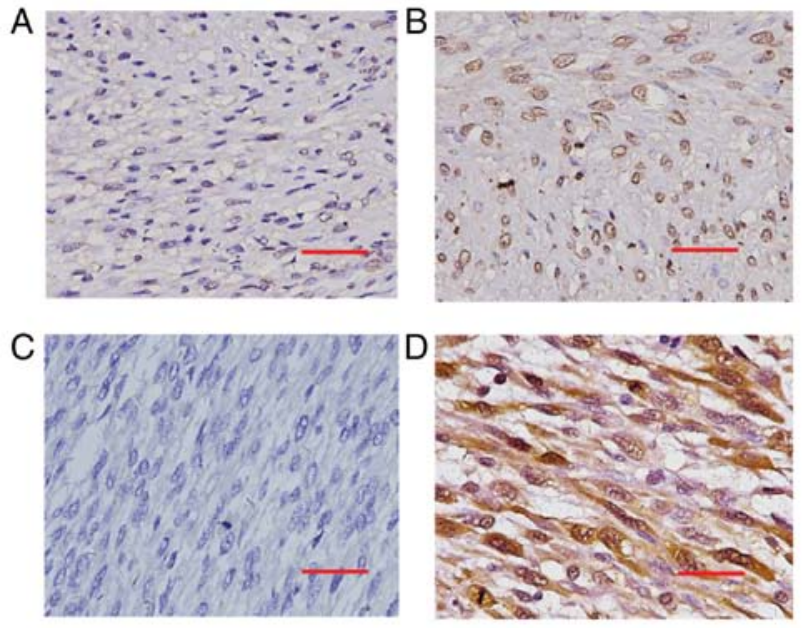

E

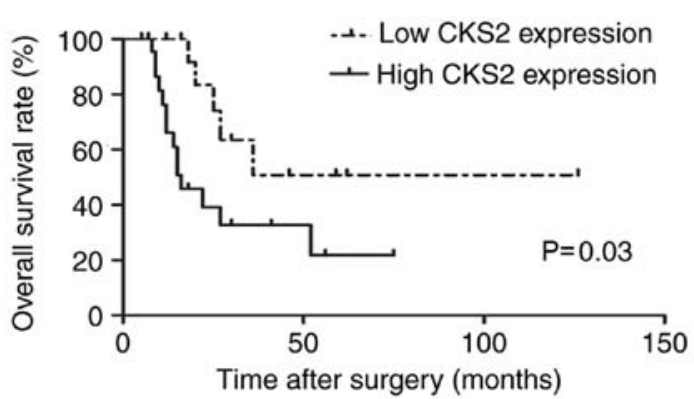

Figure 1. Elevated expression of CKS2 predicts poor prognosis in patients with ULMS. Representative images of CKS2 staining by immunohistochemistry, demonstrating (A) low expression and (B) high expression of CKS2 in ULM tissues; and (C) low expression and (D) high expression of CKS2 in ULMS tissues. Magnification, $x 400$; scale bar, $20 \mu \mathrm{m}$. (E) Kaplan-Meier survival curves of patients with ULMS according to the levels of CKS2 expression. CKS2, cyclin-dependent kinase subunit 2; ULMS, uterine leiomyosarcoma; ULM, uterine leiomyoma.

Statistical analysis. Data analysis was carried out using the SPSS 22.0 software (IBM Corp.). The association between CKS2 expression and clinicopathological factors was analyzed by Pearson's $\chi^{2}$ test. The overall survival (OS) rate was analyzed according to the Kaplan-Meier method and the generalized log-rank test was applied to analyze the survival curves. Prognostic factors were evaluated by univariate and multivariate analyses (Cox proportional hazard regression model). Other data are expressed as the mean \pm SD and analyzed with Student's t-test. All assays were performed in 
Table II. Association between CKS2 expression and clinical features of uterine leiomyosarcoma.

\begin{tabular}{|c|c|c|c|c|}
\hline \multirow[b]{2}{*}{ Parameter } & \multirow[b]{2}{*}{ Total cases, $\mathrm{n}$} & \multicolumn{2}{|c|}{ CKS2 expression } & \multirow[b]{2}{*}{ P-value } \\
\hline & & Low $(n=14), n$ & High $(n=24), n$ & \\
\hline Age, years & & & & 0.076 \\
\hline$\leq 45$ & 20 & 10 & 10 & \\
\hline$>45$ & 18 & 4 & 14 & \\
\hline Tumor size, $\mathrm{cm}$ & & & & $0.014^{\mathrm{a}}$ \\
\hline$\leq 5$ & 12 & 8 & 4 & \\
\hline$>5$ & 26 & 6 & 20 & \\
\hline FIGO stage & & & & 0.826 \\
\hline I & 26 & 11 & 15 & \\
\hline II & 3 & 1 & 2 & \\
\hline III & 5 & 1 & 4 & \\
\hline IV & 4 & 1 & 3 & \\
\hline ER expression & & & & 0.391 \\
\hline Positive & 13 & 6 & 7 & \\
\hline Negative & 25 & 8 & 17 & \\
\hline PR expression & & & & $0.048^{\mathrm{a}}$ \\
\hline Positive & 14 & 8 & 6 & \\
\hline Negative & 24 & 6 & 18 & \\
\hline
\end{tabular}

${ }^{\mathrm{a}} \mathrm{P}<0.05$. CKS2, cyclin-dependent kinases subunit 2; FIGO, International Federation of Obstetrics and Gynecology; ER, estrogen receptor; $\mathrm{PR}$, progesterone receptor.

triplicate. $\mathrm{P}<0.05$ was considered to indicate a statistically significant difference.

\section{Results}

CKS2 is overexpressed in ULMS and predicts poor prognosis. To determine the expression of CKS2 in ULMS, its protein expression was analyzed by IHC. The results indicated that the staining of CKS2 was significantly stronger in ULMS tissues than that in ULM. CKS2 expression was high in $63.2 \%$ (24 of 38 cases) of the ULMS tissues, whereas only $18.4 \%$ (7 of 38 cases) of ULM tissues had high CKS2 expression $(\mathrm{P}<0.001$; Table I; Fig. 1A-D). The expression of CKS2 was mainly located in the nucleus.

To investigate the potential roles of CKS2 in ULMS, patients were divided into two groups according to CKS2 expression. The statistical analysis demonstrated that high expression of CKS2 in patients was associated with larger tumor size and low expression of progesterone receptor (PR); whereas no association of CKS2 expression was observed with other clinicopathological features, such as age, FIGO stage and estrogen receptor expression (Table II).

The association between CKS2 expression and prognosis was determined by analyzing the OS rates of 38 patients with ULMS. As shown in Fig. 1E, patients in the CKS2 high-expression group had a markedly poorer survival rate than those in the low-expression group $(\mathrm{P}=0.03)$. Univariate analysis revealed that CKS2 expression $(\mathrm{P}=0.040)$, tumor size $(\mathrm{P}=0.045)$ and FIGO stage $(\mathrm{P}=0.001)$ were significant risk factors for OS. In multivariate analysis, CKS2 expression
$(\mathrm{P}=0.036)$ and FIGO stage $(\mathrm{P}=0.001)$ were independent predictors of OS with ULMS (Table III). Taken together, the above results indicate that $\mathrm{CKS} 2$ expression is upregulated in ULMS, which predicts poor prognosis in ULMS.

Silencing of CKS2 inhibits cell proliferation. CKS2 may contribute to the malignant behaviors of ULMS cells, because an association was found between $\mathrm{CKS} 2$ expression and tumor size. To determine the role of CKS2 in ULMS, two cell lines (SK-UT-1 and SK-UT-1B), were selected for further investigation. As shown in Fig. 2A and B, the expression of CKS2 significantly decreased, following the transfection with si-CKS2 (both $\mathrm{P}<0.01$ ). The CCK-8 assay was used to determine the change in cell viability, as shown in Fig. 2C and D, where knockdown of CKS2 decreased the viability of SK-UT-1 and SK-UT-1B cells. These results indicate that silencing of CKS2 may inhibit cell proliferation in ULMS cell lines.

Silencing of CKS2 inhibits cell cycle progression. As CKS2 is a member of the cell cycle-dependent protein kinase subunits family, cell cycle analysis was performed. The effect of CKS2 knockdown on the distribution of cells at the different phases of the cell cycle was observed. The silencing of CKS2 increased the population of cells at the $G_{1}$ phase and decreased the cells at $\mathrm{S}$ phase (Fig. $2 \mathrm{E}$ and $\mathrm{F}$ ). These results suggest that the silencing of CKS2 can cause cell cycle arrest at the $\mathrm{G}_{1} / \mathrm{S}$ transition phase in ULMS cells.

Silencing of CKS2 inhibits ULMS cell colony formation. The role of CKS2 in cell transformation was subsequently 
Table III. Univariate and multivariate analysis for prognostic factors of survival of uterine leiomyosarcoma.

\begin{tabular}{|c|c|c|c|c|c|c|}
\hline \multirow[b]{2}{*}{ Parameter } & \multirow[b]{2}{*}{ Category } & \multirow[b]{2}{*}{ Cases, n } & \multicolumn{2}{|c|}{ Univariate analysis } & \multicolumn{2}{|c|}{ Multivariate analysis } \\
\hline & & & HR $(95 \% \mathrm{CI})$ & P-value & HR $(95 \% \mathrm{CI})$ & P-value \\
\hline CKS2 expression & Low vs. high & 14 vs. 24 & $2.940(1.052-8.012)$ & $0.040^{\mathrm{a}}$ & $3.124(1.079-9.048)$ & $0.036^{\mathrm{a}}$ \\
\hline Age, years & $\leq 45$ vs. $>45$ & 20 vs. 18 & $1.144(0.463-2.825)$ & 0.771 & & \\
\hline Tumor size, $\mathrm{cm}$ & $\leq 5$ vs. $>5$ & 12 vs. 26 & $3.572(1.028-12.414)$ & $0.045^{\mathrm{a}}$ & & \\
\hline FIGO stage & I vs. II vs. III vs. IV & 26 vs. 3 vs. 5 vs. 4 & $1.949(1.312-2.896)$ & $0.001^{\mathrm{b}}$ & $2.087(1.341-3.247)$ & $0.001^{\mathrm{b}}$ \\
\hline ER expression & Positive vs. negative & 13 vs. 25 & $0.374(0.122-1.143)$ & 0.084 & & \\
\hline PR expression & Positive vs. negative & 14 vs. 24 & $0.407(0.135-1.231)$ & 0.111 & & \\
\hline
\end{tabular}

${ }^{\mathrm{a}} \mathrm{P}<0.05 ;{ }^{\mathrm{b}} \mathrm{P}<0.01$. CI, confidence interval; CKS2, cyclin-dependent kinases subunit 2; ER, estrogen receptor; FIGO, International Federation of Obstetrics and Gynecology; HR, hazard ratio; PR, progesterone receptor.

A
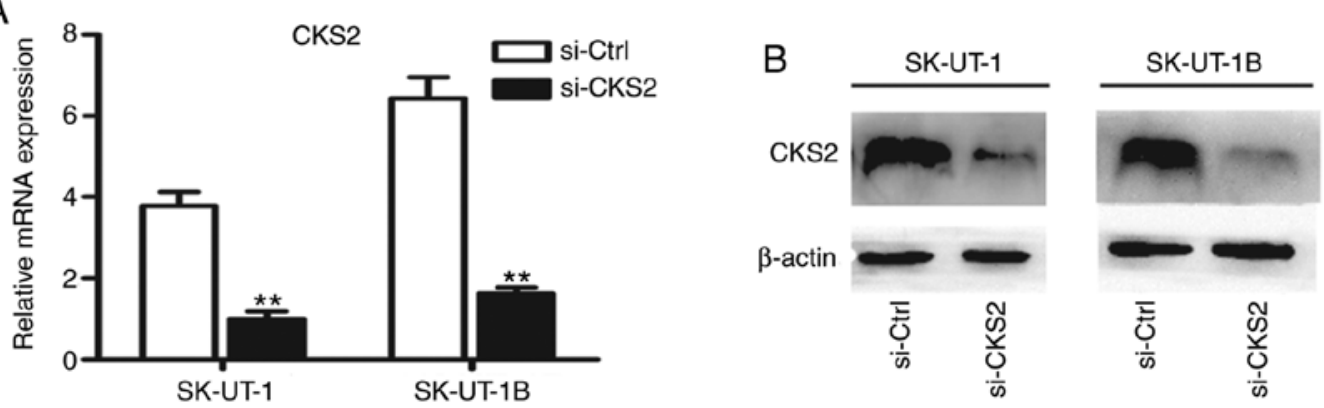

C

SK-UT-1

D

SK-UT-1B
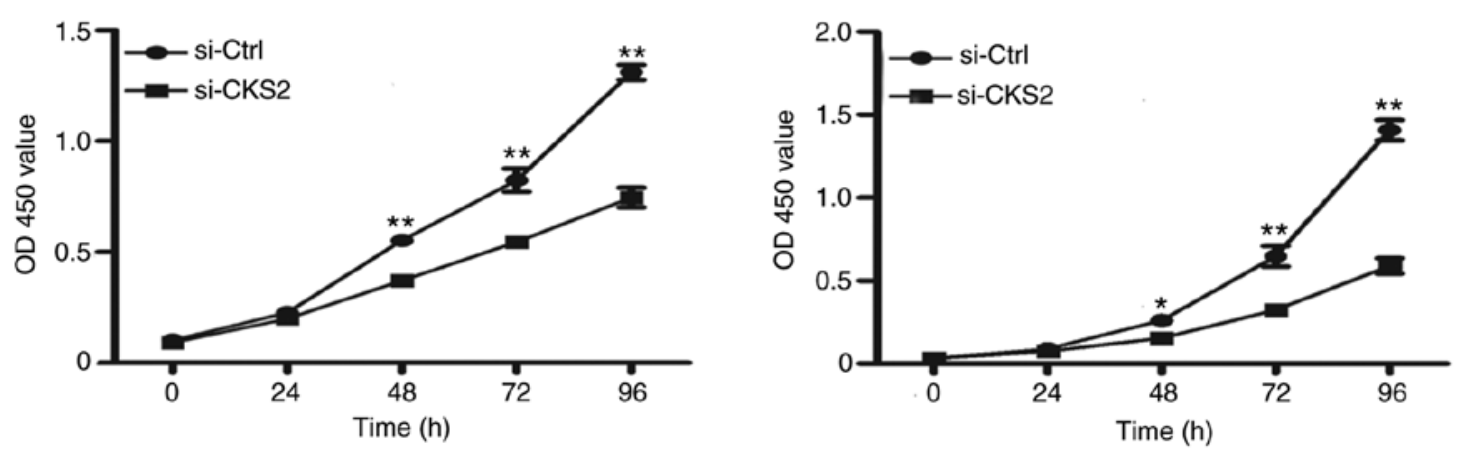

E

SK-UT-1

F

SK-UT-1B
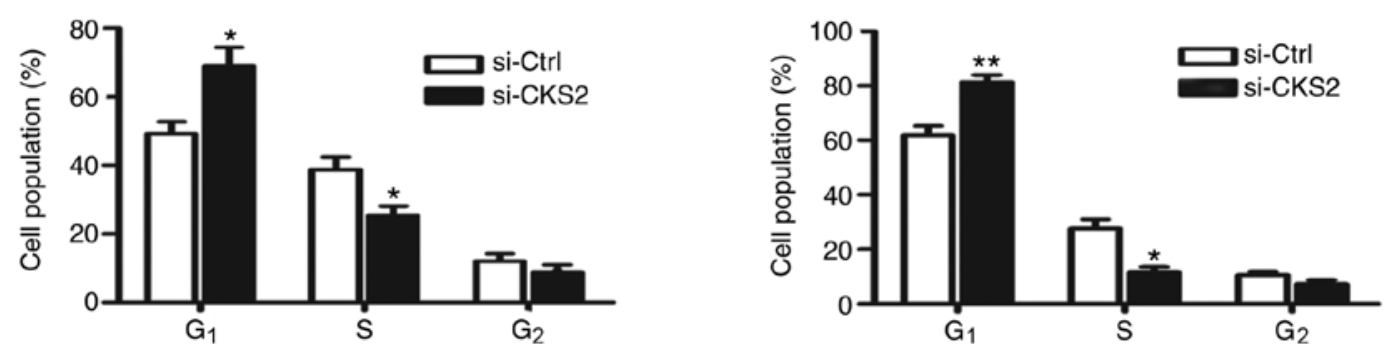

Figure 2. Suppression of CKS2 inhibits cell proliferation and cell cycle progression. (A) Reverse transcription-quantitative PCR and (B) western blot analysis of CKS2 expression to test the efficiency of si-CKS2 transfection in ULMS cells (SK-UT-1 and SK-UT-1B). Effects of CKS2 knockdown on (C and D) cell proliferation and (E and F) cell cycle in SK-UT-1 and SK-UT-1B cells. "P<0.05 and ${ }^{* *} \mathrm{P}<0.01$ vs. si-Ctrl. CKS2, cyclin-dependent kinase subunit 2; si-Ctrl, control small interfering RNA; si-CKS2, small interfering RNA targeting CKS2; OD, optical density.

examined by colony-formation assay, to analyze the oncogenic potential of CKS2 in vitro. Both of the ULMS cell lines showed diminished ability to form foci when CKS2 was downregulated by si-CKS2 (Fig. 3A). This indicated that 

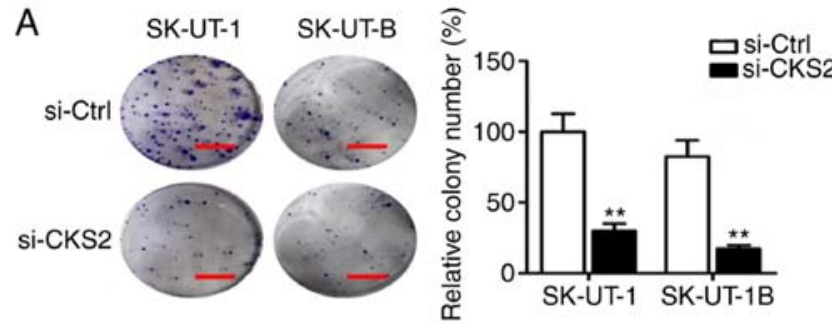

B

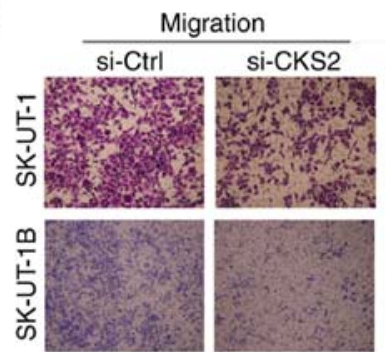

C

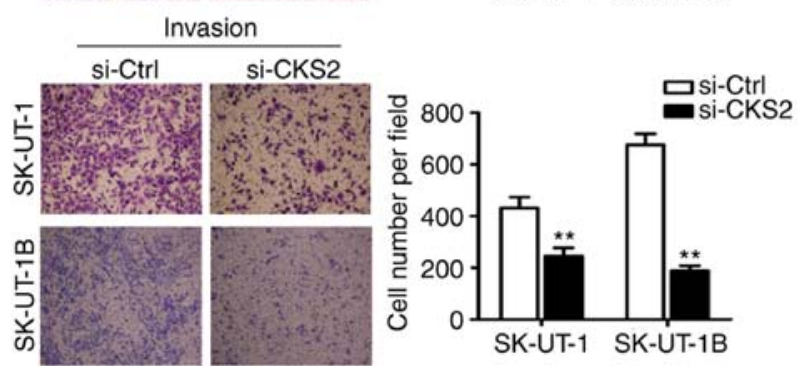

Figure 3. Silencing of CKS2 inhibits colony formation, migration and invasion in SK-UT-1 and SK-UT-1B cells. (A) The effect of CKS2 knockdown on colony formation. Scale bar, $1 \mathrm{~cm}$. (B) The effect of CKS2 knockdown on cell migration assessed by Transwell assays. (C) The effect of CKS2 knockdown on cell invasion was assessed by Matrigel-coated Transwell assays Magnification, $x 200 .{ }^{* *} \mathrm{P}<0.01$ vs. si-Ctrl. CKS2, cyclin-dependent kinase subunit 2; si-Ctrl, control small interfering RNA; si-CKS2, small interfering RNA targeting CKS2.

CKS2 contributes to promoting the clonogenic survival of ULMS cells.

Silencing of CKS2 inhibits cell migration and invasion. To further determine the role of CKS2 in the metastasis of ULMS, the migratory and invasive potential of SK-UT-1 and SK-UT-1B cells was assessed. Cells were treated with si-CKS2 or si-Ctrl and investigated in in vitro migration and Matrigel invasion assays. As shown in Fig. 3B and C, the migrating and invading cells in the si-CKS2 group were significantly decreased compared with those in the si-Ctrl group in both ULMS cell lines. These results indicate that CKS2 affects ULMS cell migration and invasion.

Furthermore, the effect of si-CKS2 on the apoptosis of ULMS cells was investigated, by measuring the apoptotic rates of si-Ctrl and si-CKS2 by Annexin V-FITC/PI double-staining assay. However, the apoptotic rate of si-CKS2 cells $(8.2 \%)$ was not significantly higher than si-Ctrl cells (5.9\%). These results suggest that CKS2 may have no effect on ULMS cell apoptosis (data not shown).

To investigate the molecular mechanisms of the effect of CKS2 in ULMS, western blot analysis was performed to measure changes in the levels of claudin-1, p-p38 and Bax, which were reported to be modulated by CKS2 in other tumors $(13,14,16,22)$. However, there were no significant changes following the silencing of CKS2 in ULMS cells (data not shown). These results suggest that CKS2 may exert its oncogenic function in ULMS through different pathways and further research is required.

\section{Discussion}

ULMS, the most common uterine sarcoma, has a poor outcome and a high recurrence rate, according to a survey from 2009 in Norway (4). Furthermore, it remains a clinical challenge to distinguish benign ULM from ULMS (23). Previous studies have shown that ULMS is characteristic of excessive activation of cell proliferation pathways and loss of chromosomal fragments that contain tumor suppressor genes $(24,25)$. The Cancer Genome Atlas found that the most commonly mutated genes of ULMS were TP53, retinoblastoma transcriptional corepressor 1, and alpha thalassemia/mental retardation syndrome X-linked (26). However, the exact pathophysiological mechanism of ULMS is still unclear.

CKS2, a member of the CKS family, was reported to be highly expressed in various malignant tumors, including breast cancer, esophageal carcinoma and gastric cancer (11-13). It was found to regulate the cell cycle and promote cancer invasion and metastasis (27). Nevertheless, the role of CKS2 in ULMS is not clearly understood.

In the present study, it was demonstrated that CKS2 was upregulated in ULMS tissues compared with ULM tissues. This was consistent with the results reported in studies by Wang et al (12) and Shen et al (15), where CKS2 was found to be upregulated in esophageal carcinoma and hepatocellular carcinoma. Moreover, Tanaka et al (13) also found that overexpression of CKS2 was correlated with tumor size, serosal invasion, lymph node metastasis and distant metastasis in gastric cancer. Furthermore, Wang et al (11) found that CKS2 was upregulated in breast cancer and associated with large tumor size, poor tumor differentiation and survival. In the present study, in addition to the association with large tumor size and poor prognosis in ULMS, CKS2 overexpression was also associated with lack of PR expression. Due to the low incidence rate of ULMS (only 0.4 out of 100,000 women each year, according to a survey from 2012 in Nordic countries) (28), the sample size is a limitation of the present study and will be addressed in future studies.

To further determine the role of CKS2 in ULMS, its expression was silenced using siRNA. The CCK-8 and colony-formation assays demonstrated that the silencing of CKS2 decreased cell viability and weakened the colony-forming ability of ULMS cells. These results indicate that CKS2 promotes cell proliferation and the clonogenic survival of ULMS cells.

Flow cytometry analysis indicated that the silencing of CKS2 in ULMS cells increased the cells at the $\mathrm{G}_{1}$ phase and decreased the cells at the $S$ phase. Yu et al (14) demonstrated that downregulation of CKS2 resulted in cell cycle arrest in $\mathrm{G}_{1} / \mathrm{S}$ transition in colorectal cancer, whereas Shen et al (16) found that silencing of CKS2 increased the number of cells at the $G_{2} / M$ phase and decreased the number at $G_{1}$ and $S$ phase in cholangiocarcinoma. CKS2 was reported to be associated with somatic cell division and meiosis $(9,10)$; however, the effect of CKS2 on the cell cycle is still unclear. In the present study, the results demonstrated that CKS2 serves as a cell 
cycle checkpoint protein for $\mathrm{G}_{1} / \mathrm{S}$ transition, which may be how CKS2 contributes to ULMS progression.

CKS2 does not only regulate the cell cycle in ULMS cells, but also has a notable effect on the capacity for migration and invasion. Specifically, the results showed that the knockdown of CKS2 inhibited ULMS cell migration and invasion in vitro. Similarly, Tanaka et al (13) and Yu et al (14) demonstrated that downregulation of CKS2 weakened the capacity of migration and invasion in gastric cancer and colorectal cancer. These findings indicate that CKS2 promotes the migration and invasion of ULMS cells, and therefore promotes metastasis. This may explain the occurrence of early metastasis in patients with ULMS. However, further study is required to elucidate the potential mechanisms.

In conclusion, this is the first study to investigate the function of CKS2 in ULMS. The findings of this study indicate that CKS2 plays an important role in ULMS and may serve as a useful marker for the differential diagnosis of ULMS and ULM. It is postulated that CKS2 exerts its oncogenic effects by promoting $\mathrm{G}_{1} / \mathrm{S}$ transition, proliferation, colony formation, migration and invasion in ULMS cells. It may also act as an independent prognostic factor in patients with ULMS and serve as a novel target for ULMS therapy.

\section{Acknowledgements}

Not applicable.

\section{Funding}

The present study was supported by the National Natural Science Foundation of China (grant no. 81372810).

\section{Availability of data and materials}

The datasets generated during the study are available from the corresponding author on reasonable request.

\section{Authors' contributions}

TZ conceived the experiments. YD and $\mathrm{QH}$ carried out the experiments and drafted the manuscript. SM, HL and FY collected the clinical data and tissue samples. JW and SG performed the statistical analysis. XJ and HX helped performing the experiments and writing the manuscript. All authors read and approved the final manuscript.

\section{Ethics approval and consent to participate}

The present study was approved by the Ethics Committee of Shandong University (approval no. 201302036). All patients provided informed written consent prior to the investigation.

\section{Patient consent for publication}

Not applicable.

\section{Competing interests}

The authors declare that they have no competing interests.

\section{References}

1. Hensley ML, Barrette BA, Baumann K, Gaffney D, Hamilton AL, Kim JW, Maenpaa JU, Pautier P, Siddiqui NA, Westermann AM and Ray-Coquard I: Gynecologic cancer intergroup (GCIG) consensus review: Uterine and ovarian leiomyosarcomas. Int J Gynecol Cancer 24 (Suppl 3): S61-S66, 2014.

2. Giuntoli RL II, Metzinger DS, DiMarco CS, Cha SS, Sloan JA, Keeney GL and Gostout BS: Retrospective review of 208 patients with leiomyosarcoma of the uterus: Prognostic indicators, surgical management, and adjuvant therapy. Gynecol Oncol 89: 460-469, 2003.

3. Kapp DS, Shin JY and Chan JK: Prognostic factors and survival in 1,396 patients with uterine leiomyosarcomas: Emphasis on impact of lymphadenectomy and oophorectomy. Cancer 112: 820-830, 2008.

4. Abeler VM, Røyne O, Thoresen S, Danielsen HE, Nesland JM and Kristensen GB: Uterine sarcomas in Norway. A histopathological and prognostic survey of a total population from 1970 to 2000 including 419 patients. Histopathology 54: 355-364, 2009.

5. Gockley AA, Rauh-Hain JA and del Carmen MG: Uterine leiomyosarcoma: A review article. Int J Gynecol Cancer 24: 1538-1542, 2014.

6. Miller H,Ike C,Parma J, Masand RP,Mach CM and Anderson ML: Molecular targets and emerging therapeutic options for uterine leiomyosarcoma. Sarcoma 2016: 7018106, 2016.

7. Richardson HE, Stueland CS, Thomas J, Russell P and Reed SI: Human cDNAs encoding homologs of the small p34Cdc28/Cdc2-associated protein of Saccharomyces cerevisiae and Schizosaccharomyces pombe. Genes Dev 4: 1332-1344, 1990.

8. Ganoth D, Bornstein G, Ko TK, Larsen B, Tyers M, Pagano M and Hershko A: The cell-cycle regulatory protein Cks1 is required for $\mathrm{SCF}(\mathrm{Skp} 2)$-mediated ubiquitinylation of $\mathrm{p} 27$. Nat Cell Biol 3: 321-324, 2001.

9. Martinsson-Ahlzén HS, Liberal V, Grünenfelder B, Chaves SR, Spruck CH and Reed SI: Cyclin-dependent kinase-associated proteins Cks1 and Cks2 are essential during early embryogenesis and for cell cycle progression in somatic cells. Mol Cell Biol 28: 5698-5709, 2008

10. Spruck CH, de Miguel MP, Smith AP, Ryan A, Stein P, Schultz RM, Lincoln AJ, Donovan PJ and Reed SI: Requirement of cks 2 for the first metaphase/anaphase transition of mammalian meiosis. Science 300: 647-650, 2003.

11. Wang J, Xu L, Liu Y, Chen J, Jiang H, Yang S and Tan H: Expression of cyclin kinase subunit 2 in human breast cancer and its prognostic significance. Int J Clin Exp Pathol 7: 8593-8601, 2014.

12. Wang JJ, Fang ZX, Ye HM, You P, Cai MJ, Duan HB, Wang F and Zhang ZY: Clinical significance of overexpressed cyclin-dependent kinase subunits 1 and 2 in esophageal carcinoma. Dis Esophagus 26: 729-736, 2013.

13. Tanaka F, Matsuzaki S, Mimori K, Kita Y, Inoue H and Mori M: Clinicopathological and biological significance of CDC28 protein kinase regulatory subunit 2 overexpression in human gastric cancer. Int J Oncol 39: 361-372, 2011.

14. Yu MH, Luo Y, Qin SL, Wang ZS, Mu YF and Zhong M: Up-regulated CKS2 promotes tumor progression and predicts a poor prognosis in human colorectal cancer. Am J Cancer Res 5: 2708-2718, 2015.

15. Shen DY, Fang ZX, You P, Liu PG, Wang F, Huang CL, Yao XB, Chen ZX and Zhang ZY: Clinical significance and expression of cyclin kinase subunits 1 and 2 in hepatocellular carcinoma. Liver Int 30: 119-125, 2010.

16. Shen DY, Zhan YH, Wang QM, Rui G and Zhang ZM: Oncogenic potential of cyclin kinase subunit-2 in cholangiocarcinoma. Liver Int 33: 137-148, 2013.

17. Kawakami K, Enokida H, Tachiwada T, Gotanda T, Tsuneyoshi K, Kubo H, Nishiyama K, Takiguchi M, Nakagawa $M$ and Seki N: Identification of differentially expressed genes in human bladder cancer through genome-wide gene expression profiling. Oncol Rep 16: 521-531, 2006

18. Walker RA: World Health Organization classification of tumours. Pathology and genetics of tumours of the breast and female genital organs. Histopathology 46: 229-229, 2005.

19. Prat J: FIGO staging for uterine sarcomas. Int J Gynaecol Obstet 104: 177-178, 2009. 
20. Coley HM, Shotton CF, Kokkinos MI and Thomas H: The effects of the CDK inhibitor seliciclib alone or in combination with cisplatin in human uterine sarcoma cell lines. Gynecol Oncol 105: 462-469, 2007.

21. Livak KJ and Schmittgen TD: Analysis of relative gene expression data using real-time quantitative PCR and the 2(-Delta Delta C(T)) method. Methods 25: 402-408, 2001.

22. Qi J, Yu Y, Akilli Öztürk Ö, Holland JD, Besser D, Fritzmann J, Wulf-Goldenberg A, Eckert K, Fichtner I and Birchmeier W: New Wnt $/ \beta$-catenin target genes promote experimental metastasis and migration of colorectal cancer cells through different signals. Gut 65: 1690-1701, 2016.

23. Cui RR, Wright JD and Hou JY: Uterine leiomyosarcoma: A review of recent advances in molecular biology, clinical management and outcome. BJOG 124: 1028-1037, 2017.

24. Cuppens T, Tuyaerts S and Amant F: Potential therapeutic targets in uterine sarcomas. Sarcoma 2015: 243298, 2015.
25. Taylor BS, Barretina J, Maki RG, Antonescu CR, Singer S and Ladanyi M: Advances in sarcoma genomics and new therapeutic targets. Nat Rev Cancer 11: 541-557, 2011.

26. Mäkinen N, Aavikko M, Heikkinen T, Taipale M, Taipale J, Koivisto-Korander R, Bützow R and Vahteristo P: Exome sequencing of uterine leiomyosarcomas identifies frequent mutations in tp53, atrx, and med12. PLoS Genet 12: e1005850, 2016.

27. You H, Lin $\mathrm{H}$ and Zhang Z: CKS2 in human cancers: Clinical roles and current perspectives (Review). Mol Clin Oncol 3: 459-463, 2015.

28. Koivisto-Korander R, Martinsen JI, Weiderpass E, Leminen A and Pukkala E: Incidence of uterine leiomyosarcoma and endometrial stromal sarcoma in Nordic countries: Results from NORDCAN and NOCCA databases. Maturitas 72: 56-60, 2012.

(i) $($ ) This work is licensed under a Creative Commons Attribution-NonCommercial-NoDerivatives 4.0 International (CC BY-NC-ND 4.0) License. 